VOL. 40 (1989) [163-174]

\title{
GROUPS WHOSE THREE-GENERATOR SUBGROUPS ARE FREE
}

\author{
Gilbert Baumslag and Peter B. Silalen
}

\section{Dedicated to Professor B.H. Neumann on his eightieth birthday}

\begin{abstract}
We define a certain class of groups, $\mathcal{C}_{k}$, which we show to contain the class of all $k$-free groups. Our main theorem shows that certain amalgamated free products of groups in $\mathcal{C}_{3}$ are again in $\mathcal{C}_{3}$. In the appendix we show that many 3 -manifold groups belong to $\mathcal{C}_{k}$ for suitable $\boldsymbol{k}$.
\end{abstract}

Let $k$ be a cardinal number. A group $G$ is said to be $k$-free if for any set $S \subseteq G$ with cardinality $k$, the subgroup of $G$ generated by $S$ is free (of some rank $\leqslant k$ ). Note that a $k$-free group is in particular $k^{\prime}$-free for every $k^{\prime} \leqslant k$. A group is 1 -free if and only if it is torsion-free.

It follows from a result proved by $B$. Baumslag in [1] that a free product with amalgamation $G=F *_{C} F^{\prime}$, where the factors $F$ and $F^{\prime}$ are free groups and the amalgamated subgroup $C$ is a maximal cyclic subgroup of each factor, is 2 -free. It is a consequence (Corollary 4.2) of the main theorem of this paper that such a group $G$ is in fact 3-free. Our proof makes strong use of the structure theorem $[6,11]$ for subgroups of free products with amalgamation, which was not available when [1] was written.

Our results apply to a broader class of groups than the one described above. Indeed, it follows from Corollary 4.1 below that if a group $G$ can be built up from free groups by repeatedly using the operation of forming a free product with an amalgamated subgroup which is a maximal cyclic subgroup of each factor, then $G$ is 3 -free. Furthermore, this remains true if the operations of forming a free product and an ascending union are also allowed.

These results are proved by defining a natural class of 3-free groups which includes free groups and is closed under the operations mentioned above. (Some evidence that our class is indeed "natural" will be offered in the appendix to this paper.)

In order to describe the appropriate class of groups, we begin by recalling some standard definitions.

If a group $H$ is given by a finite presentalion, the deficiency of the presentation is defined to be the integer $m-n$, where $m$ is the number of generators in the presentation

Received 23 June 1989

The research of both authors was partially supported by the National Science Foundation.

Copyright Clearance Centre, Inc. Serial-fee code: 0004-9729/89 \$A2.00+0.00. 
and $n$ is the number of relations. The deficiency of an arbitrary presentation of $H$ is bounded above by the torsion-free rank of the commutator quotient of $H$. The deficiency of a finitely-presented group $H$ is the largest integer that can occur as the deficiency of a finite presentation for $H$.

A group $H$ is said to be freely indecomposable if (i) $H$ is not trivial or infinite cyclic, and (ii). $H$ is not a free product of two non-trivial subgroups.

For every integer $k$ we define a class $\mathcal{C}_{k}$ of groups as follows: a group $G$ belongs to the class $\mathcal{C}_{k}$ if and only if every finitely-generated, freely indecomposable subgroup of $G$ is finitely-presented and has deficiency at least $k$. For $k \leqslant k^{\prime}$ we have $\mathcal{C}_{k} \subseteq \mathcal{C}_{k^{\prime}}$. For any $k$, a group isomorphic to a subgroup of a group in $\mathcal{C}_{k}$ is in $\mathcal{C}_{k}$, and an ascending union of groups in $\mathcal{C}_{k}$ is in $\mathcal{C}_{k}$. Any free group lies in $\mathcal{C}_{k}$ for every $k$, since a free group contains no freely indecomposable subgroup.

We now turn to some less obvious properties of the classes $\mathcal{C}_{k}$.

Proposition 1. For any non-negative integer $k$, the free product of an arbitrary family of groups in $\mathcal{C}_{k}$ is itself in $\mathcal{C}_{k}$.

Proof: Lct $G=*_{i \in I} G_{i}$ be a free product of groups in $\mathcal{C}_{k}$. Let $H$ be a finitelygenerated, freely indecomposable subgroup of $G$. (In particular $H$ is not infinite cyclic.) It follows from the Kurosh subgroup theorem that $H$ is conjugate to a subgroup of one of the $G_{i}$. Hence $H$ is finitely-presented with deficiency $\geqslant k$.

The following alternative description of the class $\mathcal{C}_{k}$ will be useful.

Proposition 2. A group $G$ belongs to $\mathcal{C}_{k}$ if and only if for every finitely-generated subgroup $H$ of $G$, either (i) $H$ is free of some rank $<k$, or (ii) $H$ is finitely-presented and has deficiency at least $k$.

Proof: The sufficiency of the stated condition is clear, since a free group is not freely indecomposable. Conversely, suppose that $G$ belongs to the class $\mathcal{C}_{k}$, and let $H$ be any finitely-generaled subgroup of $G$. It is a siandard corollary to Gruško's theorem that $H$ can be decomposed as a free product $r * H_{1} * \ldots * H_{n}$, where $n$ is a non-negative integer, $F$ is a free group of rank $m<\infty$, and each $H_{i}$ is a freely indecomposable group. Since $G \in \mathcal{C}_{k}$, each $H_{i}$ is finitely-presented and has deficiency $d_{i} \geqslant k$. Hence $H$ is finitely-presented, and the definition of deficiency implies that the deficiency of $H$ is at least $m+\sum_{i=1}^{n} d_{i}$. Hence condition (ii) of the proposition holds unless $n=0$ and $m<k$, in which case (i) holds.

A group $G$ is said to be coherent if every finitely-generated subgroup of $G$ is finitely-presented. Proposition 2 at once yields:

COROllary 2.1. If a group belongs to $\mathcal{C}_{k}$ for some $k$, then it is coherent. 
Proposition 3. Let $k$ be a non-negative integer. Then any group in the class $\mathcal{C}_{k}$ is $k$-free.

Proor: Let $G$ be a group in $\mathcal{C}_{k}$, and let $H$ be a subgroup generated by $k$ elements of $G$. By Proposition 2, either $H$ is free of rank $<k$ or $H$ has a finite presentation with deficiency $\geqslant k$. But according to a theorem due to Magnus [7], if a group $I I$ can be generated by $k$ elements and has a presentation of deficiency at least $k$, then $I I$ is free of rank $k$. Thus in any case $I$ is free.

We can now state our main result and derive its principal consequences.

TheоRem 4. Let $G=K *_{C} K^{\prime}$ be a free product with amalgamation. Suppose that $K$ and $k^{\prime}$ belong to $\mathcal{C}_{3}$ and that $C$ is a maximal cyclic subgroup of both $K$ and $K^{\prime}$. Then $G \in \mathcal{C}_{3}$.

Before proving Theorem 4 we shall point out some corollaries.

Corollary 4.1. Let $\mathcal{C}$ denote the snallest class of groups with the following properties:

(i) every free group is in $\mathcal{C}$, and the free product of any two groups in $\mathcal{C}$ is itself in $\mathcal{C}$;

(ii) any group isomorphic to a subgroup of a group in $\mathcal{C}$ is itself in $\mathcal{C}$, and any ascending union of groups in $\mathcal{C}$ is itself in $\mathcal{C}$;

(iii) if $G=K *_{C} K^{\prime}$ is a free product with amalgamation such that $K$ and $K^{\prime}$ belong to $\mathcal{C}$ and $C$ is a maximal cyclic subgroup of both $K$ and $K^{\prime}$, then $G$ is in $\mathcal{C}$.

Then every group in $\mathcal{C}$ is 3 -free.

Proof: It follows from Proposition 1, the remarks preceding Proposition 1, and Theorem 4, that conditions (i) - (iii) of the statement of the corollary hold if $\mathcal{C}$ is replaced by $\mathcal{C}_{3}$. Hence $\mathcal{C} \subseteq \mathcal{C}_{3}$. But every group in $\mathcal{C}_{3}$ is 3 -free by Proposition 3 .

Note that the proof of Corollary 4.1 also shows that every group in the class $\mathcal{C}$ is coherent. (One uses Corollary 2.1 in place of Proposition 3.) The fact that the groups in $\mathcal{C}$ are coherent is not a new result; it is contained in a more general result due to Karrass and Solitar [0, Theorem 8]. Our prouf that every group in $\mathcal{C}$ is coherent is essentially the same as the one in $[6]$.

As a special case of Corollary 4.1, we have:

Conollary 4.2. If $G=F *_{C} F^{\prime}$ is a free product with amalgamation such that $F$ and $F^{\prime \prime}$ are free groups and $C$ is a maximal cyclic subgroup of both $F$ and $F^{\prime}$, then $G$ is 3-free. 
As we indicated above, the proof of Theorem 4 uses the structure theorem for subgroups of free products with annalgamation $[0,11]$. We have found it convenient to use this theorem in the form presented by Bass and Serre in [11]. We shall begin by reviewing some elementary notions from $[11]$.

First recall that a graph $\Gamma$ is defined by two sets $V=V_{\Gamma}$ and $\vec{E}=\vec{E}_{\Gamma}$, two maps $I=I_{\Gamma}, T=T_{\Gamma}: \vec{E} \rightarrow V$, and a fixed-point free involution $\sigma=\sigma_{\Gamma}$ of $\vec{E}$ such that $I \circ \sigma=T$ and $T \circ \sigma=I$. The elements of $V$ and $\vec{E}$ are called vertices and oriented edges. For any $\vec{e} \in \vec{E}$ we call $I(\vec{e})$ and $T(\vec{e})$ the initial point and terminal point of $\vec{e}$. The orbits of $\sigma$ are called edges. We shall write $e$ for the underlying edge $\{\vec{e}, \sigma(\vec{e})\}$ of the oriented edge $\vec{e} ;$ we call $e$ and $\sigma(e)$ the orientations of $e$. The vertices $I(\vec{e})$ and $T(\vec{e})$ are the endpoints of $e$. If $I(\vec{e})=T(\vec{e})$ we call $e$ a loop. We shall let $E(\Gamma)$ denote the set of all edges of $\Gamma$.

A subgraph $\Gamma^{\prime \prime}$ of $\Gamma$ is delermined by subsets $V^{\prime}$ and $\vec{E}^{\prime}$ of $V$ and $\vec{E}$ such that $\sigma(\vec{E})=\vec{E}$ and $T(\vec{E}) \cup I(\vec{E}) \subseteq V^{\prime}$.

The graph $\Gamma$ is connected if for any $v, w \in V$ there exist $\overrightarrow{e_{1}}, \ldots, \overrightarrow{e_{n}} \in \vec{E}$ such that $I\left(\overrightarrow{e_{1}}\right)=v, T\left(\overrightarrow{e_{n}}\right)=w$, and $T\left(\overrightarrow{e_{i}}\right)=I\left(e_{i+1}\right)$ for $i=1, \ldots, n-1$. Any graph is the disjoint union of its maximal connected subgraphs, called its connected components.

A graph of groups is a pair $(\Gamma, \mathcal{G})$, where $\Gamma$ is a connected graph and $\mathcal{G}$ is a function that assigns to each vertex or edge $c$ of $\Gamma$ a group $\mathcal{G}(c)$, and to each oriented edge $\vec{e}$ a monomorphism $j^{\vec{e}}: \mathcal{G}(e) \rightarrow \mathcal{G}(T(\vec{e}))$. Note that any subgraph $A$ of $\mathrm{I}$ inherits the structure of a graph of groups $(A, \mathcal{G} \mid A)$.

As in [11], we assign to a graph of groups $(\Gamma, \mathcal{G})$ a group $\pi_{1}(\Gamma, \mathcal{G})$, its fundamental group. We shall recall some properties of $\pi_{1}(\Gamma, \mathcal{G})$ that characterise it.

If $\Gamma$ consists of a single vertex $v$ then $\pi_{1}(\Gamma, \mathcal{G})=\mathcal{G}(v)$. For any graph of groups $(\Gamma, \mathcal{G})$ and any subgraph $A$ of $\Gamma$ there is a monomorphism from $\pi_{1}(A, \mathcal{G})$ to $\pi_{1}(\Gamma, \mathcal{G})$, canonically defined modulo inner automorphisms of $\pi_{1}(\Gamma, \mathcal{G})$. In parlicular, for each vertex of $\Gamma$ there is a monomorphism $\lambda=\lambda_{v}^{\Gamma}: \mathcal{G}(v) \rightarrow \pi_{1}(\Gamma, \mathcal{G})$ which is canonical modulo inner automorphisms of $\pi_{1}(\Gamma, \mathcal{G})$.

If $e$ is an edge of $\Gamma$ we can describe $\pi_{1}(\Gamma, \mathcal{G})$ as follows. Let $\overrightarrow{e_{1}}$ and $\overrightarrow{e_{2}}$ denote the two orientations of $e$, and set $a_{i}=T\left(\overrightarrow{e_{i}}\right)$. Let $A$ denote the graph obtained from $\Gamma$ by removing the edge $e$. If $A$ is disconnected then it has two connected components $A_{1}$ and $A_{2}$, where $a_{i} \in A_{i}$; in this case $\pi_{1}(\Gamma, \mathcal{G})$ is a free product with amalgamation $\pi_{1}\left(A_{1}, \mathcal{G} \mid A_{1}\right) *_{\mathcal{G}(e)} \pi_{1}\left(A_{2}, \mathcal{G} \mid A_{2}\right)$, where $\mathcal{G}(e)$ maps to $\pi_{1}\left(A_{i}, \mathcal{G} \mid A_{i}\right)$ by the natural monomorphism $\lambda_{a_{i}} \circ j \vec{j}^{\overrightarrow{e_{i}}}$. Likewise, if $A$ is connected then $\pi_{1}(\Gamma, \mathcal{G})$ is an HNN extension $\pi_{1}(A, \mathcal{G} \mid A) *_{\mathcal{O}(e)}$, where $\mathcal{G}(e)$ maps to $\pi_{1}(A, \mathcal{G} \mid A)$ by the two monomorphisms $\lambda_{a_{i}} \circ j \overrightarrow{e_{i}}, i=1,2$. 
For a finite graph $\Gamma$ the above facts can be applied recursively to give a description of $\pi_{1}(\Gamma, \mathcal{G})$. (It is slown in [11] that the group so described is uniquely defined.) If $\Gamma$ is an infinite graph then the groups $\pi_{1}(A, \mathcal{G})$, where $A$ ranges over the finite subgraphs of $\Gamma$, form a direct system in which the maps are monomorphisms; we may then recover $\pi_{1}(\Gamma, \mathcal{G})$ as the direct limit of this systern.

If $(\Gamma, \mathcal{G})$ is a graph of groups and $e$ is an edge of $\Gamma$, there is a monomorphism $\lambda_{e}=\lambda_{e}^{\Gamma}: \mathcal{G}(e) \rightarrow \pi_{1}(\Gamma, \mathcal{G})$ given by $\lambda_{e}=\lambda_{T(\vec{e})} \circ j \vec{e}$, where $\vec{e}$ is an orientation of $e$; like the maps $\lambda_{v}$, this map is defined only modulo inner automorphisms, but it is readily seen to be independent of the choice of the orientation $\vec{e}$.

A subgroup of $\pi_{1}(\Gamma, \mathcal{G})$ will be called a vertex group or edge group if it is conjugate to a subgroup of the form $\lambda_{v}(\mathcal{G}(v))$ or $\lambda_{e}(\mathcal{G}(e))$.

In the proof of Theorem 4 we shall need the following construction.

Let $(\Gamma, \mathcal{G})$ be a finite graph of groups and let $\overrightarrow{e_{1}}$ be an oriented edge of $\Gamma$. Set $\overrightarrow{e_{0}}=\sigma\left(\overrightarrow{e_{1}}\right), v_{0}=I\left(\overrightarrow{e_{0}}\right)$ and $v_{1}=T\left(\overrightarrow{e_{0}}\right)$. Let us suppose that (i) $v_{0} \neq v_{1}$ and (ii) $j^{\overrightarrow{\vec{e}_{1}}}$ maps $\mathcal{G}\left(e_{1}\right)$ isomorphically onto $\mathcal{G}\left(v_{1}\right)$. We shall express these conditions by saying that $\overrightarrow{E_{1}}$ is contractible. Note that we have a monomorphism $\ell=j \overrightarrow{e_{0}}$ 。 $\left(j^{\overrightarrow{e_{1}}}\right)^{-1}: \mathcal{G}\left(v_{1}\right) \rightarrow \mathcal{G}\left(v_{0}\right)$.

We can obtain a connected graph $\Gamma^{\prime}$ from $\Gamma$ by "contracting" the edge $e_{1}$ in the following sense. We define $V_{\Gamma^{\prime}}$ to be the quotient of the set $V_{\Gamma}$ obtained by identifying the two elements $v_{0}$ and $v_{1}$. We write $p: V_{\Gamma} \rightarrow V_{\Gamma^{\prime}}$ for the projection map and set $v_{0}^{\prime}=p\left(v_{0}\right)$. We set $\vec{E}_{\Gamma^{\prime}}=\vec{E}_{\Gamma}-\left\{\overrightarrow{e_{1}}, \sigma\left(\overrightarrow{e_{1}}\right)\right\}, I_{\Gamma^{\prime}}=p \circ I_{\Gamma}, T_{\Gamma^{\prime}}=p \circ T_{\Gamma}$ and $\sigma_{\Gamma^{\prime}}=\sigma_{\Gamma} \mid \vec{E}_{\Gamma^{\prime}}$. Note that $E\left(\Gamma^{\prime}\right) \subset E(\Gamma)$. Next, we define a graph of groups $\left(\Gamma^{\prime}, \mathcal{G}^{\prime}\right)$. For each $e \in E\left(\Gamma^{\prime}\right) \subset E(\Gamma)$ we set $\mathcal{G}^{\prime}(e)=\mathcal{G}(e)$. We define $\mathcal{G}^{\prime}\left(v_{0}^{\prime}\right)$ to be $\mathcal{G}^{\prime}\left(v_{0}\right)$. If $v^{\prime} \in V\left(\Gamma^{\prime}\right)-\left\{v_{0}^{\prime}\right\}$ then $v^{\prime}=p(v)$ for a unique $v \in V\left(\Gamma^{\prime}\right)$; in this case we set $\mathcal{G}^{\prime}(v)=\mathcal{G}(v)$. If $\vec{e} \in \vec{E}_{\Gamma^{\prime}} \subset \vec{E}_{\Gamma}$ and $T(\vec{e}) \neq v_{1}$ we set $j_{\mathcal{g}^{\prime}} \overrightarrow{e^{\vec{e}}}=j_{\mathcal{g}}$. On the other hand, if $\vec{e} \in \vec{E}_{\Gamma^{\prime}}$ and $T(\vec{e})=v_{1}$ then we set $j_{\mathcal{g}^{\prime}}^{\vec{e}}=\ell \circ j_{g}^{\vec{e}}$.

The graph of groups $\left(I^{\prime \prime}, \mathcal{G}^{\prime}\right)$ is said to be obtained from $(\Gamma, \mathcal{G})$ by contracting $\overrightarrow{e_{1}}$. The gist of the following lemma is that this operation does not change the fundamental group.

LEMMA 5. Let $\overrightarrow{e_{1}}$ be a contractible edge in a finite graph of groups $(\Gamma, \mathcal{G})$. Let $\left(\Gamma^{\prime}, \mathcal{G}^{\prime}\right)$ be obtained from $(\Gamma, \mathcal{G})$ by contracting $\overrightarrow{e_{1}}$. Let $p: V_{\Gamma} \rightarrow V_{\Gamma^{\prime}}$ denote the projection map. Then there is an isomorphism $J: \pi_{1}(\Gamma, \mathcal{G}) \rightarrow \pi_{1}\left(\Gamma^{\prime}, \mathcal{G}^{\prime}\right)$ such that, modulo inner automorphisms of $\pi_{1}\left(I^{\prime \prime}, \mathcal{G}^{\prime}\right)$, we have

(i) $J \circ \lambda_{v}^{\Gamma}=\lambda_{p(v)}^{\Gamma^{\prime}}$ for every $v \in V_{\Gamma}$, and

(ii) $J \circ \lambda_{e}^{\Gamma}=\lambda_{e}^{\Gamma^{\prime}}$ for every $e \in E\left(\Gamma^{\prime}\right) \subset E\left(\Gamma^{\prime}\right)$. 
Proof: We argue by induction on the number of edges of $\Gamma$. First suppose that $e_{1}$ is the only edge. Since $\overrightarrow{e_{1}}$ is contractible, $e_{1}$ is not a loop; hence $\Gamma$ has exactly two vertices, $v_{0}=I\left(\overrightarrow{e_{1}}\right)$ and $v_{1}=I^{\prime}\left(\overrightarrow{e_{1}}\right)$. The group $\pi_{1}(\Gamma, \mathcal{G})$ is a free product with amalgamation $\mathcal{G}\left(v_{0}\right) *_{\mathcal{G}\left(e_{1}\right)} \mathcal{G}\left(v_{1}\right)$. Since $\overrightarrow{e_{1}}$ is contractible, $j^{\overrightarrow{e_{1}}}$ maps $\mathcal{G}\left(e_{1}\right)$ isomorphically onto $\mathcal{G}\left(v_{1}\right)$. Thus $\pi_{1}(\Gamma, \mathcal{G})$ is canonically isomorphic to $\mathcal{G}\left(v_{0}\right)$. On the other hand, $\Gamma^{\prime}$ has a single vertex $v^{\prime}$ in this case, and $\pi_{1}\left(\Gamma^{\prime}, \mathcal{G}\right)=\mathcal{G}\left(v^{\prime}\right)=\mathcal{G}\left(v_{0}\right)$. The resulting canonical isomorphism between $\pi_{1}(\Gamma, \mathcal{G})$ and $\pi_{1}\left(\Gamma^{\prime}, \mathcal{G}^{\prime}\right)$ is readily seen to satisfy (i) and (ii).

Now suppose that $\Gamma$ has more than one edge. Let $e$ be an edge different from $e_{1}$. Let $A$ be the graph obtained from $\Gamma$ by removing $e$. Consider the case where $A$ is connected. In this case $(A, \mathcal{G} \mid A)$ is a graph of groups, and $\overrightarrow{e_{1}}$ is a contractible edge of $(A, \mathcal{G} \mid A)$. Furthermore, the graph of groups obtained from $(A, \mathcal{G} \mid A)$ by contracting $\overrightarrow{\boldsymbol{e}}_{1}$ is canonically isomorphic to $\left(A^{\prime}, \mathcal{G}^{\prime} \mid A^{\prime}\right)$, where $A^{\prime}$ is the (connected) graph obtained from $I^{\prime \prime}$ by renoving the edge $e$. By the induction hypothesis there is an isomorphism $J_{A}: \pi_{1}(A, \mathcal{G} \mid A) \rightarrow \pi_{1}\left(A^{\prime}, \mathcal{G}^{\prime} \mid A^{\prime}\right)$ such that

(i $\left.{ }_{A}\right) \quad J_{A} \circ \lambda_{v}^{A}=\lambda_{p(v)}^{A^{\prime}}$ for every $v \in V_{A}$, and

(ii $\left.{ }_{A}\right) \quad J_{A} \circ \lambda_{e}^{A}=\lambda_{e}^{A^{\prime}}$ for every $e \in E\left(A^{\prime}\right) \subset E(A)$.

Now $\pi_{1}(\Gamma, \mathcal{G})$ is an HNN extension $\pi_{1}(A, \mathcal{G} \mid A) * \mathcal{G}(e)$, and $\pi_{1}\left(\Gamma^{\prime}, \mathcal{G}^{\prime}\right)$ is an HNN extension $\pi_{1}\left(A^{\prime}, \mathcal{G} \mid A^{\prime}\right) *_{\mathcal{G}(e)}$. It follows from $\left(\mathrm{i}_{A}\right)$ and $\left(\mathrm{ii}_{A}\right)$ that the isomorphism $J_{A}$ is compatible, modulo inner automorphisms of $\pi_{1}\left(A^{\prime}, \mathcal{G} \mid A^{\prime}\right)$, with the monomorphisms from $\mathcal{G}(e)$ to $\pi_{1}(A, \mathcal{G} \mid A)$ and $\pi_{1}\left(A^{\prime}, \mathcal{G} \mid A^{\prime}\right)$ involved in these HNN extensions; thus $J_{A}$ extends to an isomorphism $J: \pi_{1}(\Gamma, \mathcal{G}) \rightarrow \pi_{1}\left(\Gamma^{\prime}, \mathcal{G}^{\prime}\right)$. It is easy to check that $J$ satisfies (i) and (ii). This completes the induction in the case where $A$ is connected. If $A$ is disconnected the argument is the same, except that free products with amalgamation appear in place of HNN extensions.

'The next lemma is essentially contained in [6]; we have included an explicit proof for the sake of convenience.

Lemma 6. Let $(\Gamma, \mathcal{G})$ be a finite graph of groups. Suppose that $\mathcal{G}(e)$ is finitelygenerated for every $e \in E\left(\Gamma^{\prime}\right)$ and that $\pi_{1}(\Gamma, \mathcal{G})$ is finitely-generated. Then $\mathcal{G}(v)$ is finitely-generated for every $v \in V(\Gamma)$.

Proof: It is a special case of [6, Theorem 4] that if a free product with amalgamation $A{ }^{*} C B$ is finitely-generated and the amalgamated subgroup $C$ is finitely generated, then the factors $A$ and $B$ are finitely-generated. Similarly, by [6, Lemma 3 ], if an HNN extension $A *_{C}$ is finitely-generated and the associated subgroup $C$ is finitely-generated, then the base group $A$ is also finitely-generated. The lemma follows from these facts by virtue of the recursive definition of $\pi_{1}(\Gamma, \mathcal{G})$ given above. 
The next four lenmmas are intended to summarise information that will be needed for the proof of Theorem 4.

LEMмA 7. Let $(\Gamma, \mathcal{G})$ be a finite graph of groups in which all the vertex groups are finitely-presented and all the edge groups are cyclic. Then $\pi_{1}(\Gamma, \mathcal{G})$ is finitely-presented and its deficiency is at least $1+\sum_{v}\left(d_{v}-1\right)$, where $v$ ranges over the vertices of $\Gamma$, and where $d_{v}$ denotes the deficiency of $\mathcal{G}(v)$.

Proof: We use induction on the number $n$ of vertices of $\Gamma$. For $n=1$ the assertion is obvious. If $n>1$ then the graph $\Gamma$ has at least one edge $e$. The graph $A$ obtained by removing the edge $e$ from $\Gamma$ has one or two connected components. If $A$ has two components $A_{1}$ and $A_{2}$ then $\pi_{1}(\Gamma)$ is the free product of $\pi_{1}\left(A_{1}, \mathcal{G} \mid A_{1}\right)$ and $\pi_{1}\left(A_{2}, \mathcal{G} \mid A_{2}\right)$ with the cyclic amalgamated subgroup $\mathcal{G}(e)$. Hence the deficiency of $\pi_{1}(\Gamma, \mathcal{G})$ is at least $d_{1}+d_{2}-1$, where $d_{i}$ is the deficiency of $\pi_{1}\left(A_{i}, \mathcal{G} \mid A_{i}\right)$. The induction step follows at once in this case. If $A$ is connected then $\pi_{1}(\Gamma, \mathcal{G})$ is an HNN extension with base group $\pi_{1}(A, \mathcal{G} \mid A)$ and cyclic associated subgroup $\mathcal{G}(e)$. Hence in this case the deficiency of $\pi_{1}(\Gamma, \mathcal{G})$ is at least that of $\pi_{1}(A, \mathcal{G} \mid A)$. Again the induction step follows.

Recall that a subgroup $C$ of a group $K$ is malnormal if for every $x \in K-C$ we have $x C \boldsymbol{x}^{-1} \cap C=\{1\}$.

LEMMA 8. If $K$ is a group in the class $\mathcal{C}_{2}$, then any maximal cyclic subgroup of $K$ is malnormal.

Proof: Let $C$ be a maximal cyclic subgroup, and let $x \in K-C$ be given. Let $y$ be a generator of $C$. Since $K \in \mathcal{C}_{2}$, it follows from Proposition 3 that the subgroup $I I$ of $K$ generated by $x$ and $y$ is free of rank at most 2 . If $H$ has rank $\leqslant 1$ then it is a cyclic subgroup containing $C$; hence $I=C$. This is impossible since $x \notin C$. Hence $H$ has rank 2 and is therefore freely generated by $x$ and $y$. It follows that $x C x^{-1} \cap C=\{1\}$.

LEMMA 9. If the hypotheses of Theorem 4 hold, then $C, K$ and $K^{\prime}$ are malnormal in $G$. Furthermore, if $H$ is any finitely-generated subgroup of $G$, then $H$ is isomorphic to the fundamental group of a finite graph of groups $(\Gamma, \mathcal{G})$ with the following properties:

(i) every vertex group of $(\Gamma, \mathcal{G})$ is either a free group of rank $\leqslant 2$ or a finitely-presented group of deficiency $\geqslant 3$;

(ii) the edge groups and vertex groups of $(\mathrm{I}, \mathcal{G})$ are all malnormal in $H$;

(iii) every edge group of $(\mathrm{I}, \mathcal{G})$ is infinite cyclic or trivial.

Proof: It follows from Lemma 8 that $C$ is malnormal in $K$ and $K^{\prime}$. This implies, by a straightforward application of the normal form (see B.H. Neumann [9]) for elements 
of a free product with amalgamation, that $C, K$ and $K^{\prime}$ are malnormal in $G=K{ }_{C} K^{\prime}$.

To prove the second assertion, we regard $G$ as the fundamental group of a graph of groups in which every vertex group is conjugate to $K$ or $K^{\prime}$ and every edge group is conjugate to $C$. By [11, Theorem 12], $G$ acts on some (simplicial) tree $X$ in such a way that the stabiliser (in $G$ ) of every vertex of $X$ is conjugate to $K$ or $K^{\prime}$, and the stabiliser of every edge of $X$ is conjugate to $C$. Now let $H$ be a finitely-generated subgroup of $G$. By restricting the above action of $G$ on $X$, we obtain an action of $H$ on $X$ under which the stabiliser of every vertex of $X$ is the intersection of $H$ with a conjugate of $K$ or $K^{\prime}$ in $G$, and the stabiliser of every edge of $X$ is the intersection of $H$ with a conjugate of $C$ in $G$. Hence by $[11$, Theorem 13], $H$ is the fundamental group of a graph of groups $(\Gamma, \mathcal{G})$, where each vertex group of $\pi_{1}(\Gamma, \mathcal{G})$ is the intersection of $H$ with a conjugate of $K$ or $K^{\prime}$ in $G$, and each edge group of $\pi_{1}(\Gamma, \mathcal{G})$ is the intersection of $H$ with a conjugate of $C$ in $G$.

By the above discussion of graphs of groups, $H=\pi_{1}(\Gamma, \mathcal{G})$ is the direct limit of the groups $\pi_{1}(A, \mathcal{G})$, where $A$ ranges over the finite subgraphs of $\Gamma$; the maps in the direct system are monomorphisms. Since $H$ is finitely-generated, it follows that $H \cong \pi_{1}\left(\Gamma^{\prime}, \mathcal{G}\right)$ for some finite subgraph $\Gamma^{\prime}$ of $\Gamma$. Hence we may assume that $\Gamma$ is a finite graph.

The vertex groups of $\Gamma$ are finitely generated by Lemma 6, and are conjugate to sulogroups of $K$ or $K^{\prime}$, which belong to the class $\mathcal{C}_{3}$; thus it follows from Proposition 2 that conclusion (i) of the lemma holds. Likewise, (iii) holds because the edge groups are conjugate to subgroups of the cyclic group $C$. Finally, each vertex group or edge group in $\pi_{1}(\Gamma, \mathcal{G})$ is the intersection of $H$ with a conjugate of $K, K^{\prime}$ or $C$; since the latler subgroups are malnormal in $G$, it is clear that the vertex groups and edge groups in $\pi_{1}(\Gamma, \mathcal{G})$ are malnormal in $H$.

LEMma 10. Suppose that the hypotheses of Theorem 4 hold, and that $H$ is a finitely-generated, freely indecomposable subgroup of $G$. Then $H$ is isomorphic to the fundamental group of a finite graph of groups $\left(\Gamma_{0}, \mathcal{G}_{0}\right)$ with the following properties:

$\left(i_{0}\right)$ every vertex group of $\left(\Gamma_{0}, \mathcal{G}_{0}\right)$ is either a free group of rank 2 or a finitely presented group of deficiency $\geqslant 3$;

(iiv) the graph $\Gamma_{0}$ has no loops;

(iii $\left.{ }_{0}\right)$ every edge group of $\left(\Gamma_{0}, \mathcal{G}_{0}\right)$ is infinite cyclic.

Proof: Among all finite graphs of groups $(\Gamma, \mathcal{G})$ satisfying conditions (i) - (iii) of Lemma 9 and having fundamental group isomorphic to $H$, choose one, say $\left(\Gamma_{0}, \mathcal{G}_{0}\right)$, whose underlying graph $\Gamma_{0}$ has the smallest possible number of vertices. We claim that $(l, \mathcal{G})$ satisfies $\left(i_{0}\right)$ - (iii 0$)$.

We establish (iii $\left.{ }_{0}\right)$ first; this anounts to showing that no edge group of $\left(\Gamma_{0}, \mathcal{G}_{0}\right)$ 
is trivial. Suppose that $\mathcal{G}_{0}(e)$ is trivial for some edge $e$. The graph $A$ obtained from $\mathrm{I}_{0}$ by removing the edge $e$ has one or two connected components. If $A$ has two components $A_{1}$ and $A_{2}$, then $\pi_{1}\left(\Gamma_{0}, \mathcal{G}_{0}\right) \cong \pi_{1}\left(A_{1}, \mathcal{G}_{1}\right) * \pi_{1}\left(A_{2}, \mathcal{G}_{2}\right)$, where $\mathcal{G}_{i}=\mathcal{G}_{0} \mid A_{i}$. Since $I$ is freely indecomposable, one of the factors, say $\pi_{1}\left(A_{1}, \mathcal{G}_{1}\right)$, is trivial. But then $I I \cong \pi_{1}\left(A_{2}, \mathcal{G}_{2}\right)$; since $A_{2}$ has fewer vertices than $\Gamma_{0}$, this contradicts our choice of $\left(\Gamma_{0}, \mathcal{G}_{0}\right)$. If $A$ is connected then $\pi_{1}\left(\Gamma_{0}, \mathcal{G}_{0}\right) \cong \pi_{1}\left(A, \mathcal{G}_{0} \mid A\right) * Z$; this contradicts the hypothesis that $H$ is freely indecomposable.

Next we establish (ii ${ }_{0}$ ). If $e$ is an edge of $\Gamma_{0}$ having both its endpoinls at a vertex $v$, and $A$ is the subgraph of $\Gamma$ consisting of the vertex $v$ and the edge $e$, then $\pi_{1}\left(A, \mathcal{G}_{0} \mid A\right) \leqslant \pi_{1}\left(\Gamma_{0} \mathcal{G}_{0}\right)=H \leqslant G$ is an HNN extension with base group $\mathcal{G}_{0}(v)$ and associaled subgroup $\mathcal{G}(e)$. By (iii $\left.{ }_{0}\right), \mathcal{G}(e)$ is infinite cyclic. Hence $\mathcal{G}_{0}(v)$ is not malnormal in $H$. This contradicts condition (ii) of Lemma 9.

To prove ( $\left.i_{0}\right)$ we must show that no vertex group of $\left(\Gamma_{0}, \mathcal{G}_{0}\right)$ is infinite cyclic or trivial. Suppose that $\mathcal{G}_{0}(v)$ is trivial or cyclic for some $v$. If $v$ is not incident to any edge of $\Gamma_{0}$ then the graph $\Gamma_{0}$ consists of the vertex $v$ alone; in this case we have $H=\mathcal{G}_{0}(v)$, so that $H$ is itself cyclic or trivial. This contradicts the hypothesis that $H$ is freely indecomposable.

Now suppose that there is an edge $e$ of $\Gamma_{0}$ incident to $v$. By (ii ${ }_{0}$ ), $e$ is not a loop. By (iii $)_{0}, \mathcal{G}_{0}(e)$ is infinite cyclic; by (ii) it is malnormal. It follows that $\mathcal{G}_{0}(e)$ is a maximal cyclic sulggroup of $G$. Since $H$ is cyclic, the map $j_{e, v}: \mathcal{G}_{0}(e) \rightarrow \mathcal{G}_{0}(v)$ must be an isomorphism. Thus $e$ is a contractible edge. Let $(\Gamma, \mathcal{G})$ denote the graph of groups obtained by contracting $e$. By Lemma 5 , there is an isomorphism between $\pi_{1}(l, \mathcal{G})$ and $\pi_{1}\left(\Gamma_{0}, \mathcal{G}_{0}\right)$ that takes edge groups to edge groups. Thus $H$ can be identified isomorphically with $\pi_{1}(\Gamma, \mathcal{G})$ in such a way that condition (ii) of Lemma 9 holds. It is clear from the definition of contraction that $(\Gamma, \mathcal{G})$ also satisfies conditions (i) and (iii) of Lemma 9, and that $\Gamma$ has fewer vertices than $\Gamma_{0}$. This contradicts the definition of $\left(\Gamma_{0}, \mathcal{G}_{0}\right)$.

Proof of Theorem 4: Let $H$ be a finitely-generated, freely indecomposable sulugroup of $G$. Let us exhibit $H$ as the fundamental group of a graph of groups $\Gamma, \mathcal{G}$ satisfying conditions $\left(i_{0}\right)-\left(i i i_{0}\right)$ of lemma 10. Condition ( $\left.i_{0}\right)$ implies that each vertex group of $\Gamma, \mathcal{G}$ is finitely presented and has deficiency $\geqslant 2$. Hence if $\Gamma$ has at least two vertices, it follows from Lemma 7 (and condition (iii ${ }_{0}$ )) that $H$ has deficiency $\geqslant 3$.

Now suppose that $\Gamma$ has a single vertex $v$. By condition ( ii $_{0}$ ) of Lemma 10 it follows that $\Gamma$ has no edges, that is it consists of the vertex $v$ alone. Therefore $H=\mathcal{G}(v)$. Since $H$ is freely indecomposable, it now fullows from condition $\left(\mathrm{j}_{0}\right)$ that $H$ is finitely-presented with deficiency at least 3 . 


\section{APPENDIX}

The classes $\mathcal{C}_{k}$, for $k \in N$, seem to be of independent interest. For example, in this appendix we shall show that many 3 -manifold groups belong to $\mathcal{C}_{k}$ for suitable positive values of $k$. (That 3-inanifold groups are colierent was proved by Scott [10] and Shalen (unpublished).) As an application of our result we shall give a criterion (Corollary A1) for subgroups of 3 -manifold groups to be free; this criterion generalises the closed case of [5, VI.4.1] (see also [13]).

In the following discussion we shall work, in general, in the piecewise-linear category. A 3-manifold $M$ is irreducible if every (PL) 2-sphere in $M$ bounds a ball. (For relevant background in 3-manifold theory, see [3] or [4].)

For any integer $g \geqslant 0$, let $S_{g}$ denote the closed orientable surface of genus $g$. For example, $\pi_{1}\left(S_{1}\right)$ is free abelian of rank 2 .

TIEOREM A. Let $M$ be a non-compact, irreducible, orientable 3-manifold, and set $G=\pi_{1}(M)$. Let $k$ be a positive integer, and suppose that $G$ has no subgroup isomorphic to $\pi_{1}\left(S_{g}\right)$ for any $g<k$. Then $G \in \mathcal{C}_{k}$.

Proof: Let $H$ be any freely indecomposable, finitely-generated subgroup of $G$. By elementary covering space theory, $H$ is isomorphic to the fundamental group of some covering space $\widetilde{M}$ of $M$. Since $M$ is non-compact, so is $\widetilde{M}$. According to the sphere theorem of Papakyriakopoulos [3, Chapter 4], the irreducibility of $M$ implies that $\pi_{2}(M)=0$. Hence $\pi_{2}(\widetilde{M})=0$.

By [10], the manifold $\widetilde{M}$ has a compact core, that is, it contains a compact (connected) manifold-with-boundary $N \subset \widetilde{M}$ such that the inclusion $N \hookrightarrow \widetilde{M}$ induces an isomorphism of fundamental groups. Since $\widetilde{M}$ is non-compact, $N$ has non-empty boundary.

Among all compact cores for $\widetilde{M}$, let us suppose $N$ to be chosen so as to have the smallest possible number of boundary components. Then no component of $N$ is a sphere. (Indeed, since $\pi_{2}(\widetilde{M})=0$, any spliere component $S$ of $\partial N$ would bound a compact, simply-connected submanifold $B$ of $\widetilde{M}:$ see $[8$, Proof of Theorem 2]. Since $N$ is connected we would have $N \subset B$ or $N \cap B=S$. But $N \subset B$ would imply that $\pi_{1}(N)=\{1\}$, contradicting the free indecomposability of $H=\pi_{1}(N)$. If we had $N \cap B=S$, the manifold $N^{\prime}=N \cup B$ would be a compact core for $\widetilde{M}$ having fewer boundary components than $N$.)

We claim that for every component $X$ of $\partial N$, the inclusion $X \hookrightarrow N$ induces a monomorphism of fundamental groups. lndeed, if the induced homomorphisin $\pi_{1}(X) \hookrightarrow$ $\pi_{1}(N)$ has a non-trivial kernel, then by the loop theorem of Papakyriakopoulos [3, Chapter 4], some non-trivial element of the kernel is represented by a simple closed 
curve $\gamma \subset X$, and $\gamma$ bounds an enubedded disk $D$ in $N$ with $\partial D=D \cap \partial N$. If $N-D$ is connected, it follows from van Kampen's theorem that $\pi_{1}(N) \cong \pi_{1}(N-D) * \mathrm{Z}$; this contradicts the free indecomposability of $H \cong \pi_{1}(N)$. If $N-D$ is not connected, it has two components $A$ and $B$. Since $\gamma$ is homotopically non-trivial in $\partial N$, each of the manifolds $A$ and $B$ has a positive-genus boundary component; hence $\pi_{1}(A)$ and $\pi_{1}(B)$ are both non-trivial. But van Kampen's theorem gives $\pi_{1}(N) \cong \pi_{1}(A) * \pi_{1}(B)$, and again the free indecomposability of $I I$ is contradicled. This proves the clairn.

Thus for every component $X$ of $\partial M$, the group $\pi_{1}(X)$ is isomorphic to a subgroup of $I I$ and hence to a subgroup of $G$. Since no component of $\partial N$ is a sphere, the hypothesis of the theorem implies that each component has genus at least $k$. Since $\partial N \neq \emptyset$, it follows that $\chi(\partial N) \leqslant 2-2 k$, where $\chi$ denotes Euler characteristic. By Poincaré-Lefschelz duality we have $\chi(N)=(1 / 2) \chi(\partial N) \leqslant 1-k$.

As $N$ is a compact connected 3-manifold with non-empty boundary, it is homotopyequivalent to a finite $C W$ complex $K$. After collapsing a maximal tree in the 1-skeleton of $K$ to a point, we may suppose that $K$ has a single vertex. If $m$ denotes the number of 1-cells in $K$, and $n$ the number of 2-cells, then $H \cong \pi_{1}(K)$ has a presentation with $m$ generators and $n$ relations. On the other hand, we have $1-m+n=\chi(K)=\chi(N)=$ $(1 / 2) \chi(\partial N) \leqslant 1-k$, so that $m-n \geqslant k$. This shows that $H$ is finitely-presented and has deficiency at least $k$, and completes the proof of the theorem.

CoROllary A1. Let $G$ be the fundamental group of an irreducible, orientable 3manifold. Let $k$ be a positive integer, and suppose that $G$ has no subgroup isomorphic to $\pi_{1}\left(S_{g}\right)$ for any $g<k$. Then any finitely-generated, freely indecomposable subgroup of infinite index in $G$ has deficiency at least $k$. In particular, any infinite-index subgroup of $G$ generated by at most $k$ elements is free (of some rank $\leqslant k$ ).

PRoof: If $G=\pi_{1}(M)$ then any $I \leqslant G$ is isomorphic to the fundamental group of some covering space $\widetilde{M}$ of $M$. If $H$ has infinite index in $G$ then $\widetilde{M}$ is non-compact, so that $I I \in \mathcal{C}_{k}$ by the theorem. Hence if $I I$ is finitely-generated and freely indecomposable then it has deficiency at least $k$. This proves the first assertion. It follows that every infinite-index subgroup of $G$ belongs to the class $\mathcal{C}_{k}$. Hence by Proposition 3, if an infinite-index subgroup of $G$ is generated by at most $k$ elements then it is free.

The case $k=2$ of Corollary A1 includes the closed case of VI.4.1 of [5]. One can also prove a version of Corollary A1 that includes the bounded case of [5, VI.4.1]; we leave the details to the reader.

Corollary A2. Let $G$ be the fundamental group of an irreducible, orientable 3-manifold $M$. Let $k$ be a positive integer, and suppose that

(i) $\operatorname{rank} H_{1}(M ; Q)=\operatorname{rank} I_{1}(G ; Q)>k$, and

(ii) $G$ has no subgroup isomorphic to $\pi_{1}\left(S_{g}\right)$ for any $g<k$. 
Then $G$ is $k$-free.

Proof: It follows from (i) that every $k$-generator subgroup of $G$ has infinite index. Hence the assertion follows from Corollary A1.

For example, $G=\pi_{1}(M)$ is 2 -free provided that $H_{1}(M ; Q)$ has rank at least 3 and $G$ contains no free abelian group of rank 2. (The latter condition holds if $M$ is hyperbolic; see [2].)

In [12] it will be shown that if $k$ is a positive integer, and $M$ is an orientable 3-manifold such that

$$
\operatorname{rank} H_{1}\left(M ; Z_{p}\right) \geqslant k+\frac{3+\sqrt{8 k+9}}{2}
$$

for some prime $p$, then any $k$ elements of $\pi_{1}(M)$ generate an infinite-index subgroup. Hence Corollary A2 remains true if (i) is replaced by (*). For example, if an orientable hyperbolic 3 -manifold $M$ satisfies rank $H_{1}\left(M ; Z_{p}\right) \geqslant 6$ for some prime $p$, then $\pi_{1}(M)$ is 2 -free. Some applications of this fact will be given in [12].

\section{REFERENCES}

[1] B. Baumslag, 'Generalized free products whose two-generator subgroups are free', J. London Math. Soc. 43 (1968), 601-606.

[2] P. Fatou, 'Fonctions automorphes', Volume 2 of Théorie des Fonctions algébriques, R.E. Appell and E. Goursat (Gauthiers-Villars, Paris). 158-160.

[3] J. Hempel, 3-manifolds: Ann. of Math. Studies 86 (Princeton University Press, 1076).

[4] W. Jaco, Lectures on 3-manifold topology (Amer. Math. Soc. for CBMS, Providence, c., 1980).

[5] W. Jaco and P.B. Shalen, Seifert fibered spaces in 3-manifolds (Mem. Amer. Math. Soc. 21, no. 220, 1979).

[6] A. Karrass and D. Solitar, 'The subgroups of a free product of two groups with an amalgamated sulsgroup', Thans. Amer. Math. Soc. 160 (1870), 227-255.

[7] W. Magnus, 'Über freie Faktorgruppen und freie Untergruppen gegebener Gruppen', Monatsh. Math. u. Phys. 47 (1939), 307-313.

[8] J. Milnor, 'A unique decomposition theoren for 3-manifolds', Amer. J. Math. 84 (1962), 1-7.

[8] B.H. Neumann, 'An essay on free product of groups with amalgamations', Philos. Trans. Roy. Soc. London Ser. A 246 (1954), 503-554.

[10] G.P. Scott, 'Compact submanifolds of 3-manifolds', J. London Math. Soc. 7 (1973), 246-250.

[11] J.-P Serre, Arbres, amalgames, $S L_{2}$, Astérisque 46: Translated as Trees (Springer-Verlag, Berlin, Heidelberg, New York, 1980).

[12] P.B. Shalen and P. Wagreich, 'Growth, volumes and free subgroups of 3-manifold groups', (In preparation).

[13] T. 'Tucker, 'On Kleinian groups and 3-manifolds of Euler characteristic zero', (Unpublished).

Department of Mathernatics

City College (CUNX)

New York, NY 10031

United Stales of America

\section{Department of Mathematics, Statistics and Computer Science}

The University of Illinois at Chicago

Chicago, IL 60680

United States of America 